\title{
dc field-emission analysis of GaAs and plasma-source ion-implanted stainless steel
}

\author{
C. Hernandez, ${ }^{\text {a) }}$ T. Wang, T. Siggins, D. Bullard, H. F. Dylla, and C. Reece \\ Jefferson Laboratory, Newport News, Virginia 23606
}

N. D. Theodore and D. M. Manos

College of William and Mary, Williamsburg, Virginia 23187

(Received 11 November 2002; accepted 31 March 2003; published 30 June 2003)

\begin{abstract}
Field-emission studies have been performed on a GaAs wafer and a sample of its stainless-steel (SS) support electrode that are part of a photocathode gun for the $10 \mathrm{~kW}$ Upgrade infrared free electron laser at Jefferson Lab. The objective of the studies presented here is to characterize the effect of both the cleanliness of the wafer and the plasma-source ion-implanted layer on the electrode to suppress field emission. Field emission is the limiting factor to achieve the required $6 \mathrm{MV} / \mathrm{m}$ at the surface of the wafer. Potential field emitters are first located on the surface of 1 in. diameter samples with a dc scanning field-emission microscope at $60 \mathrm{MV} / \mathrm{m}$, then each emitter is characterized in a scanning electron microscope equipped with an energy dispersive spectrometer. The GaAs wafer was hydrogen cleaned before the study. The results show three emitters caused by indium contamination during wafer handling. The GaAs wafer thus shows good high-voltage characteristics and the need to maintain cleanliness during handling. The SS sample is hand polished with diamond paste to a $1 \mu \mathrm{m}$ surface finish, then implanted with $\mathrm{N}_{2} / \mathrm{SiO}_{2}$ in a plasma-source ion-implantation chamber in preparation for the field-emission studies. (c) 2003 American Vacuum Society.
\end{abstract}

[DOI: $10.1116 / 1.1577131]$

\section{INTRODUCTION}

The design of the driver accelerator for the $10 \mathrm{~kW} \mathrm{Up-}$ grade of the Jefferson Lab infrared free electron laser (IR FEL) calls for a $10 \mathrm{~mA}$ continuous-wave (cw) electron beam with $135 \mathrm{pC}$ single bunch charge, and normalized emittance less than $30 \mathrm{~mm}$ mrad. ${ }^{1} \mathrm{~A}$ dc photocathode high brightness source has been fully characterized and proven to meet the specifications (5 mA cw electron-beam current, $60 \mathrm{pC}$ charge per bunch) for the previous $1 \mathrm{~kW}$ IR Demo FEL. ${ }^{2}$ In this source, the electrons are extracted from a cesium-doped GaAs bulk wafer by a frequency-doubled, mode-locked $\mathrm{Nd}$ :YLF laser at $527 \mathrm{~nm}$, and accelerated at the surface of the wafer with the highest possible electric field to minimize emittance growth due to the high charge per bunch. The wafer is mounted on a planar section of an 8 in. diameter ball cathode attached to a $33 \mathrm{in}$. long support tube. The anode is a 10 in. diameter circular plate located in front of the wafer with a central aperture for laser and electron-beam transport. All of the electrodes are fabricated from a vacuum arc remelt, cross-forged 304 stainless-steel (SS) (hereafter referred to simply as SS in this work), then hand polished with diamond paste to a $1 \mu \mathrm{m}$ surface finish to minimize field emission. Figure 1 shows a schematic of the photocathode gun including results from POISSON simulations at $500 \mathrm{kV}$ (operating gun voltage). As can be seen from Fig. 1, the electric field at the surface of the wafer is $6 \mathrm{MV} / \mathrm{m}$, while the highest field $(12.4 \mathrm{MV} / \mathrm{m})$ is in the support tube near the ground end ring. ${ }^{3}$ In these relatively high electric fields, the performance of the gun is limited by field emission from the electrode

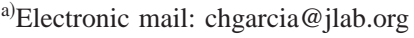

structures. In field emission, the classical potential barrier set by the work function of the metal is thinned by the presence of a strong electric field (in the $\mathrm{GV} / \mathrm{m}$ level), allowing the electrons to tunnel through from the metal to the vacuum as described by the Fowler-Nordheim equation ${ }^{4}$

$$
J=\frac{1.54 \times 10^{-6} E^{2}}{\phi} \times \exp \left[\frac{-6.83 \times 10^{9} \times \phi^{3 / 2}}{E}\right],
$$

where $J$ is the field-emission current density in $\mathrm{A} / \mathrm{m}^{2}, \phi$ is the work function of the metal in $\mathrm{eV}$, and $E$ is the local microscopic (i.e., emission site) electric field in $\mathrm{V} / \mathrm{m}$ (for $E$ $<1 \mathrm{GV} / \mathrm{m}$, field-emission current density is negligible).

Field-emitted electrons may cause sputtering of the materials inside the gun structure that may result in arcing or breakdown. Arcing may cause permanent field-emission sites on the electrodes and release gases that chemically contaminate the GaAs wafer dramatically reducing its quantum efficiency. ${ }^{2,5}$ An electron gun for the $10 \mathrm{~kW}$ Upgrade IR FEL is being assembled incorporating improvements in the operating vacuum conditions, and especially in the photocathode activation system. In the photocathode activation system of the previous gun, cesium was delivered to the wafer by means of a dispenser tube and a leak valve, ${ }^{3}$ spraying the ball cathode surface as well. This method is unfavorable in the sense that cesium lowers the work function of the metal, ${ }^{6}$ therefore, decreasing the onset electric field for field emission as seen from Eq. (1). A major improvement in the upgraded electron gun is that cesium will be delivered directly to the wafer by cesium channels mounted inside the ball cathode. To further suppress field emission, the ball cathode and its support tube have been immersed in nitrogen plasma to be ion implanted and simultaneously coated with $\mathrm{SiO}_{2}$ in 


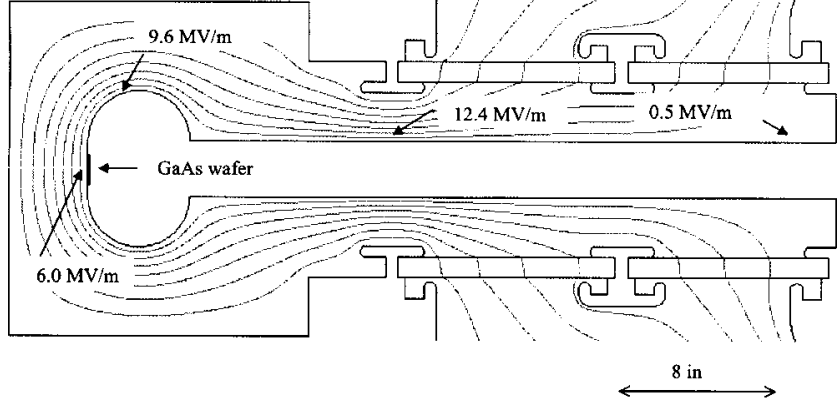

FIG. 1. Electron gun schematic with equipotential lines and field strengths calculated for $500 \mathrm{kV}$.

a plasma-source ion implantation system. This process is based on the results of previous studies that show a dramatic reduction of field-emission current from large area ionimplanted electrodes. ${ }^{5}$

The studies presented here focus on a more detailed fieldemission analysis of samples of the two materials on the surface of the gun subjected to high electric fields, bulk GaAs wafer, and hand-polished $\mathrm{N}_{2} / \mathrm{SiO}_{2}$-implanted SS electrodes. We emphasize the study of GaAs here and present some results on a comparison of field-emission studies between a bare and the $\mathrm{N}_{2} / \mathrm{SiO}_{2}$-implanted SS samples. In a companion article, ${ }^{7}$ we present more detailed characterization studies of the SS samples.

\section{EXPERIMENTAL TECHNIQUES}

The bulk GaAs wafer, which serves as the electron gun photocathode after activation by cesium deposition, is a disk $3.1 \mathrm{~cm}$ in diameter and $635 \mu \mathrm{m}$ thick. The procedure described next for this study is followed identically during the preparation of a wafer for regular operation in the FEL photocathode gun.

The GaAs wafer was first immersed in isopropanol and cleaned for $15 \mathrm{~min}$ in an ultrasonic cleaner, then mounted on a molybdenum holder at the end of a SS stalk. A piece of indium (In) foil is placed between the molybdenum holder and the wafer. The indium foil melts during the heat-cleaning cycle described next, providing a uniform contact surface that reduces the mechanical stress on the wafer and allows for a more uniform temperature distribution. The wafer was held in place with a tantalum cup after being cleaned of dust with a nitrogen flow. The stalk was then inserted into the hydrogen cleaning system utilized to prepare the GaAs wafer for its installation and regular operation in the FEL photocathode gun. ${ }^{2,8}$ Once the system had been evacuated to about $10^{-9}$ Torr, the wafer was heat cleaned at $600{ }^{\circ} \mathrm{C}$ for $15 \mathrm{~min}$ by means of a heater attached to the back of the molybdenum holder. The temperature was decreased and maintained constant at $300^{\circ} \mathrm{C}$ during the hydrogen clean cycle. Hydrogen gas was then introduced to the vacuum chamber by means of a leak valve adjusted to maintain a constant pressure of $\sim 15$ mTorr. The plasma was generated with a helical rf coil, initiating a hydrogen cleaning cycle that lasted for about $1 \mathrm{~h}$.
The heater was turned off and the wafer was cooled down overnight, removed from its holder, and placed in a nylon bag filled with nitrogen for transport.

To characterize the effect on field-emission suppression of the $\mathrm{N}_{2} / \mathrm{SiO}_{2}$-implanted gun electrodes, two samples were machined from the same type of SS used for the fabrication of electrodes. Each sample is a disk $3.1 \mathrm{~cm}$ in diameter and $0.2 \mathrm{~cm}$ in thickness. Following a similar polishing process for the actual gun electrodes, the samples were hand polished starting with sandpaper and finishing with $1 \mu \mathrm{m}$ diamond paste. The samples were ultrasonic cleaned in a three-step process. First, the samples were immersed in a degreaser soap diluted in filtered and deionized (DI) water and placed in the ultrasonic cleaning system for about $30 \mathrm{~min}$. After a thorough rinse with DI water, the samples were dipped in acetone and ultrasonic cleaned for $15 \mathrm{~min}$, and finally immersed in methanol for another $15 \mathrm{~min}$ in the ultrasonic cleaner. The samples were then from the container, allowed to dry in air, and transported in closed plastic containers to minimize dust exposure. One of the samples was taken directly to the dc scanning field-emission microscope (SFEM) described later, while the other sample was mounted inside the plasma-source ion implantation chamber.

The plasma-source ion-implantation system has been extensively described and characterized elsewhere. ${ }^{5,7,9}$ The sample was mounted on a SS electrode plate electrically insulated from ground and connected to a high-voltage (HV) pulse forming network capable of delivering up to $-100 \mathrm{kV}$ in pulses $20 \mu$ s long at adjustable repetition rates up to 200 $\mathrm{Hz}$. The chamber was then evacuated with a $1000 \mathrm{l} / \mathrm{s}$ turbomaglev pump to a final pressure of $\leqslant 10^{-6}$ Torr. Nitrogen was introduced into the chamber by means of a leak valve. The plasma was then initiated with an rf power supply coupled to the plasma by a planar coil antenna resting on a large area unshielded quartz window that seals the top of the vacuum chamber. Sputtering of the quartz window due to electrostatic coupling between the antenna and the plasma generates a constant stream of $\mathrm{SiO}_{2}$ within the chamber. By negatively biasing the sample, the nitrogen atoms in the plasma are implanted at energy comparable to the applied voltage, while at the same time, the sputtered $\mathrm{SiO}_{2}$ from the quartz window forms a uniform coating on the sample surface.

After the preparation procedures just described, all three samples; the GaAs wafer, the bare SS, and the $\mathrm{N}_{2} / \mathrm{SiO}_{2}$-implanted SS samples, were transported to a class1000 clean room for field-emission studies. Each sample was studied independently in the dc SFEM. Individual emitters on each sample were first identified with the SFEM and then individually characterized with a scanning electron microscope (SEM) equipped with an energy dispersive spectrometer (EDS). The SFEM, the SEM, and the EDS comprise the complete system currently used for field-emission studies on niobium. This system is described in detail elsewhere. ${ }^{10,11}$ Each sample was mounted in the SEM chamber, which serves as introduction chamber for the SFEM, then transferred under a vacuum to the SFEM chamber 


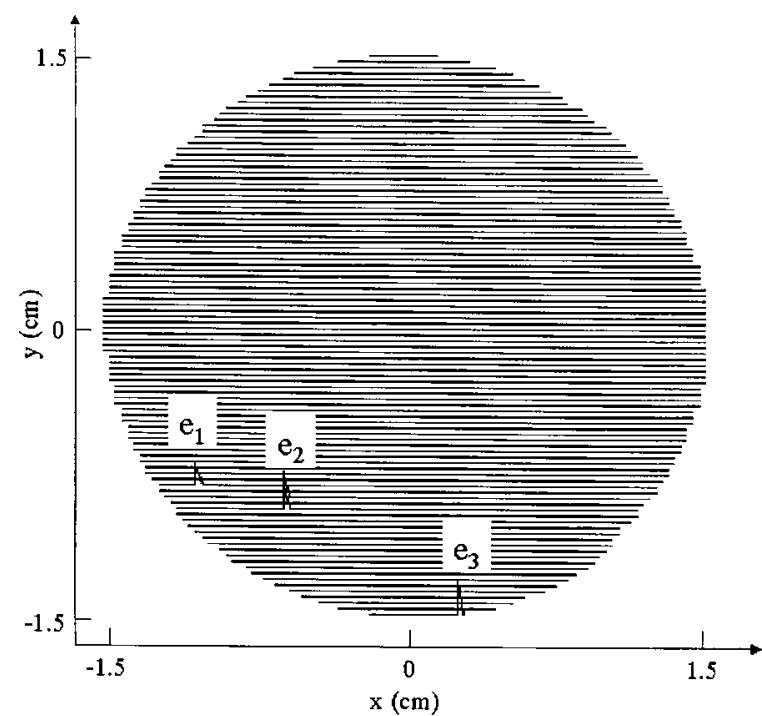

FIG. 2. Field-emission scan map shows three emitters on the surface of the bulk GaAs sample. The average scanning electric field was $60 \mathrm{MV} / \mathrm{m}$.

$\left(\sim 10^{-9}\right.$ Torr $)$ and mounted on a stage that can be moved in $x, y$, and $z$ axes by computer-controlled stepper motors. The cathode is the sample itself, while the anode is a tungsten cylinder about $2 \mathrm{~cm}$ long and $150 \mu \mathrm{m}$ in radius with a flat end tip for coarse resolution scans. A paraboloid tip $(10 \mu \mathrm{m}$ curvature radius) tungsten needle is employed for mediumresolution scans. The electric field $F$ at the cathode directly below the anode tip is $F=V / \kappa d$, where $V$ is the applied voltage to the needle, $\kappa$ is the field correction factor, and $d$ is the anode-cathode gap. ${ }^{12}$ The field-emission scan, performed every $250 \mu \mathrm{m}$ across the sample surface, started by positioning the needle at the center of the sample, then the $\mathrm{HV}$ was increased from 0 up to $35 \mathrm{kV}$, or until a threshold current $(2 \mathrm{nA})$ between the anode and the cathode was detected by a picoameter. The voltage was then recorded if that threshold current was observed, indicating the presence of an emitter. If no current was detected over the voltage range, then no emitter was found at that scan location. The two SS samples were scanned at $140 \mathrm{MV} / \mathrm{m}$ scanning field (electric field at the midpoint of the $250 \mu \mathrm{m}$ scan step) with the flattipped cylindrical anode. In this case, the curvature radius is much larger than the anode-cathode gap chosen for this experiment $(d=200 \mu \mathrm{m})$, so the electric field is simply $F$ $=V / d$.

\section{EXPERIMENTAL RESULTS AND DISCUSSION}

The GaAs sample was dc field-emission scanned in the SFEM at $60 \mathrm{MV} / \mathrm{m}$ maximum field using the $10 \mu \mathrm{m}$ paraboloid anode tip $(\kappa=1.5)$ and $d=400 \mu \mathrm{m}$ of anode-cathode gap. Figure 2 shows the resulting field-emission map, where the three emission sites found on the surface of the sample can be clearly seen. Each peak marks the location of each emission site. The height of each peak is represented by

$$
h=C\left[V_{0}-V(x, y)\right] \text {, }
$$

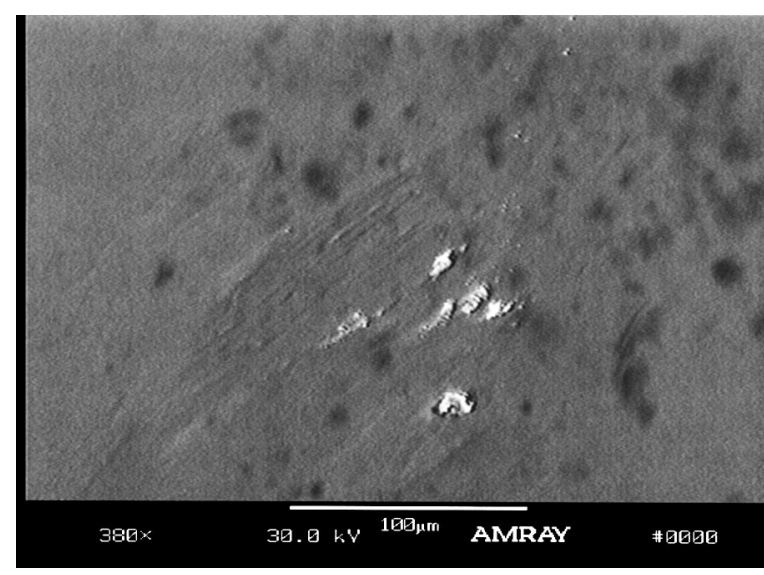

FIG. 3. SEM picture of emitter $1\left(\mathrm{e}_{1}\right)$ on the surface of the GaAs sample. The white traces are contaminants on the GaAs surface identified as indium by the EDS spectrum.

where $C$ is a constant, $V_{0}$ is the maximum applied voltage $(35 \mathrm{kV})$, and $V(x, y)$ is the highest voltage before reaching the threshold current at coordinates $(x, y) .{ }^{11}$ Therefore, the pattern on the map is flat $(h=0)$ in the absence of emitters $\left[V_{0}=V(x, y)\right]$, while the taller the peak at a given emission site, the lower the onset electric field for field emission. The onset electric field for the emitters labeled as $\mathrm{e}_{1}, \mathrm{e}_{2}$, and $\mathrm{e}_{3}$ in Fig. 2 is $54 \mathrm{MV} / \mathrm{m}, 47 \mathrm{MV} / \mathrm{m}$, and $30 \mathrm{MV} / \mathrm{m}$, respectively. After identifying the emission sites with the aid of an optical microscope mounted on the SFEM, the sample was transported under a vacuum to the SEM chamber to relocate and characterize each individual emitter. The white features seen on the SEM picture of $\mathrm{e}_{1}$ in Fig. 3 were identified as indium by the EDS apparatus. Similar results were obtained for $\mathrm{e}_{2}$ and $e_{3}$. As a manner of calibration, a spectrum was taken on several featureless locations across the sample. As expected, only gallium $(\mathrm{Ga})$ and arsenic (As) were identified as the components of the bulk wafer. The indium traces can therefore be characterized as contaminants on the GaAs surface, most likely transferred by the tools employed in the process of mounting the wafer onto its molybdenum holder.

Even though the onset electric field for field emission of the foreign emitters is at least a factor of 5 above the operating electric field at the surface of the GaAs wafer, it must be noted that the field-emission current can be started at much lower fields once the wafer is activated (as a photocathode) by cesium deposition. Cesium lowers the work function of the GaAs bulk wafer (as well as metals with different work functions) ${ }^{6}$ from $5.5 \mathrm{eV}$ down to $1.5 \mathrm{eV}{ }^{13}$ therefore, increasing the field-emission current, as can be seen from Eq. (1).

The bare SS sample was scanned using the flat anode tip at an average scanning electric field of $140 \mathrm{MV} / \mathrm{m}(\kappa=1$ and $d=200 \mu \mathrm{m})$. The SFEM scan showed ten emitters for this particular sample. The onset electric field for field emission varies from $105 \mathrm{MV} / \mathrm{m}$ to $175 \mathrm{MV} / \mathrm{m}$. The EDS spectrum taken on one of the features shows the same elements characteristic of the bare SS sample (approximately 70\% Fe, $20 \% \mathrm{Cr}$, and $10 \% \mathrm{Ni}$ plus small traces of $\mathrm{C}, \mathrm{Mn}, \mathrm{Si}, \mathrm{P}$, and 


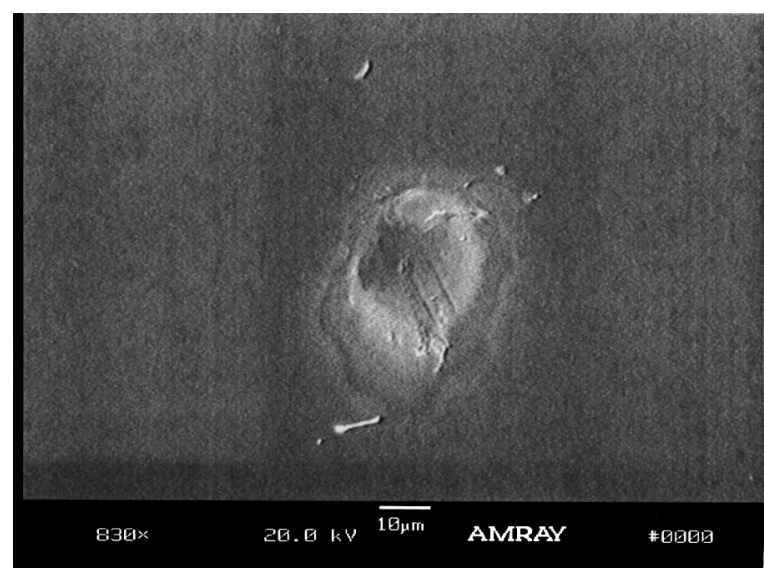

FIG. 4. SEM picture of emitter $\mathrm{e}_{1}$ on the $\mathrm{N}_{2} / \mathrm{SiO}_{2}$-implanted SS sample. It is possible that unipolar arcing during the ion source plasma implantation process might have caused the crater seen in the picture.

S), indicating that the emitter is either a fragment of the sample broken off from the surface during polishing that was not removed by the cleaning techniques described in Sec. II, or a basin that was not polished off completely.

The field-emission scan for the $\mathrm{N}_{2} / \mathrm{SiO}_{2}$-implanted SS sample was performed under the same parameters as those for the bare SS sample. The resulting field-emission map shows only two emission sites (compared to ten found on the bare SS sample). The onset electric field for field emission is $135 \mathrm{MV} / \mathrm{m}$ for emitter $1\left(\mathrm{e}_{1}\right)$ and $115 \mathrm{MV} / \mathrm{m}$ for emitter 2 $\left(e_{2}\right)$. Figure 4 is an SEM picture of $e_{1}$. The EDS spectrum taken on a featureless location of the sample shows the characteristic peaks of SS including the peak for silicon. At the time that the samples were analyzed, the EDS apparatus was not able to detect elements lighter than fluorine; therefore, the expected peak for oxygen (as part of the $\mathrm{SiO}_{2}$ layer) was not identified. The EDS spectrum taken on the center of the crater seen on the picture shows the expected SS component, but no silicon. However, the spectrum shows a high intensity silicon peak on the crater edge, as well as on the strandlike feature seen on the lower left-hand side of the crater. The splattered material around the crater seen in Fig. 4 and the exposed SS at its center revealed by the EDS point to unipolar arcing as a probable mechanism for the formation of this kind of emitter, ${ }^{14,15}$ which could have been initiated by a fragment of SS. Unipolar arcing has been observed previously on large area test samples during the course of the present work. Table I summarizes the results from the three studied samples.

TABLE I. SFEM and EDS results for each sample.

\begin{tabular}{cccc}
\hline \hline Sample & $\begin{array}{c}\text { No. of } \\
\text { emitters }\end{array}$ & $\begin{array}{c}\text { Onset electric } \\
\text { field (MV/m) }\end{array}$ & $\begin{array}{c}\text { EDS emitter } \\
\text { composition }\end{array}$ \\
\hline GaAs & 3 & $30-54$ & In \\
Bare SS & 10 & $100-170$ & $\mathrm{SS}$ \\
Implanted SS & 2 & 115 and 135 & $\mathrm{SS}+\mathrm{Si}$ \\
\hline \hline
\end{tabular}

\section{CONCLUSIONS}

The field-emission studies presented here show that the surface of the GaAs wafer can sustain more than the $6 \mathrm{MV} / \mathrm{m}$ required for the photocathode gun operation. Our cleaning techniques were aimed to remove surface contaminants and not material. However, it is crucial to have a dedicated set of tools for handling the indium foil to minimize the presence of foreign particles that become potential field emitters after the wafer activation by cesium deposition. Even though the onset electric field for field emission of the foreign particles (indium, in this case) was found to be at least five times higher than the operating surface electric field at the wafer, the work function of metals decreases to levels low enough to activate field emission at moderate electric fields.

The results on the bare SS sample show that $1 \mu \mathrm{m}$ finish polishing alone removes potential field emitters with an onset electric field for field emission below $100 \mathrm{MV} / \mathrm{m}$, which is about ten times higher than the highest calculated field on the surface of the ball cathode support tube. However, one must consider that the area of the samples studied here is about 100 times smaller than the area of the test electrodes mentioned in Ref. 2 (and even smaller than the surface area of the gun electrodes) where inclusions and surface defects (both potential field emitters) are more likely to occur just by the large surface area.

The onset electric field for field emission for the few emitters found in the $\mathrm{N}_{2} / \mathrm{SiO}_{2}$-implanted SS sample did not increase significantly. However, the reduction of the total number of emitters (by a factor of 5) with respect to bare SS is certainly encouraging toward the operation of the plasmasource ion-implanted electrodes for the $10 \mathrm{~kW}$ Upgrade photocathode gun of the Jefferson Lab IR FEL.

\section{ACKNOWLEDGMENTS}

This work was supported by the U.S. Dept. of Energy, the Office of Naval Research, the Air Force Research Laboratory, the Commonwealth of Virginia, and the Laser Processing Consortium.

${ }^{1}$ D. Douglas, S. V. Benson, G. A. Krafft, R. Li, L. Merminga, and B. C. Yunn, Proceedings of the 20th International Linac Conference, Monterey, CA, 2000, p. 857.

${ }^{2}$ T. Siggins et al., Nucl. Instrum. Methods Phys. Res. A 475, 549 (2001).

${ }^{3}$ D. A. Engwall, Ph.D. thesis, University of Illinois at Urbana-Champaign, 1998.

${ }^{4}$ R. H. Fowler and L. Nordheim, Proc. R. Soc. London, Ser. A 119, 173 (1928).

${ }^{5}$ C. K. Sinclair, H. F. Dylla, T. L. Siggins, D. Manos, L. Wu, and T. J. Venhaus, Proceedings of the 2001 Particle Accelerator Conference, Chicago, IL, p. 610.

${ }^{6}$ N. D. Lang, Phys. Rev. B 4, 4234 (1971).

${ }^{7} \mathrm{~N}$. D. Theodore et al., these proceedings.

${ }^{8}$ C. K. Sinclair, B. M. Poelker, and J. S. Price, Proceedings of the 1997 Particle Accelerator Conference, Vancouver, British Columbia, 1997, p. 2864.

${ }^{9}$ L. Wu, Ph.D. thesis, College of William and Mary, 2000.

${ }^{10} \mathrm{~T}$. Wang, C. Reece, and R. Sundelin, Proceedings of the Tenth SRF Workshop, Tsukuba, Japan, 2001.

${ }^{11}$ T. Wang, C. E. Reece, and R. M. Sundelin, Rev. Sci. Instrum. 73, 3215 (2002). 
${ }^{12}$ P. Nierdermann, Ph.D. thesis, University of Geneva, 1986.

${ }^{13}$ H. Gao, J. Vac. Sci. Technol. A 5, 1295 (1987).

${ }^{14}$ A. V. Nedospasov, V. G. Petrov, and N. M. Zykova, IEEE Trans. Plasma Sci. 13, 253 (1985).
${ }^{15}$ G. A. Mesyats, Proceedings of the PPPS-2001 Pulsed Power Plasma Science 2001, 28th IEEE International Conference on Plasma Science, and 13th IEEE International Pulsed Power Conference, Las Vegas, NV, 2001, p. 165. 